\title{
Impaired Platelet Procoagulant Mechanisms in Patients with Bleeding Disorders
}

Harvey J. Weiss, M.D. ${ }^{1,2}$

The publisher regrets an error in Table 1 in the above article in Seminars in Thrombosis and Hemostasis, Volume 35, Number 2, 2009, p. 235.

Table 1 with the correct wording in column three (PCA-Related Defect(s)) appears below.

Table 1 Impaired Platelet PCA in Bleeding Disorders

\begin{tabular}{|c|c|c|c|c|}
\hline Disorder & $\begin{array}{l}\text { Primary Platelet } \\
\text { Defect(s) }\end{array}$ & $\begin{array}{l}\text { PCA-Related } \\
\text { Defect(s) }\end{array}$ & $\begin{array}{l}\text { Other Platelet } \\
\text { Defects }\end{array}$ & $\begin{array}{l}\text { Screening PCA } \\
\text { Abnormality }\end{array}$ \\
\hline Scott syndrome & $\begin{array}{l}\text { PS exposure, } \\
\text { vesiculation }\end{array}$ & $\begin{array}{c}\text { Factor } \mathrm{Va}, \mathrm{Xa}, \mathrm{IX} \text { a binding, } \\
\text { prothrombinase, tenase }\end{array}$ & (See text) & SPT, PF3a \\
\hline Platelet vesiculation defect & Vesiculation & Vesiculation & None & SPT \\
\hline Quebec platelet disorder & uPA overexpression & $\begin{array}{l}\text { Prothrombinase, } \\
\text { platelet factor } \mathrm{V}\end{array}$ & $\begin{array}{l}\text { Proteolyzed } \alpha \text {-granule } \\
\text { proteins }\end{array}$ & \\
\hline Platelet factor V-New York & Platelet factor $\mathrm{V}$ & Prothrombinase & None & PF3a \\
\hline$\delta$-Storage pool deficiency & Dense granules & $\begin{array}{l}\text { Prothrombinase, ATP, ADP } \\
\text { secretion, } \mathrm{Ca}^{2+} \text { entry }\end{array}$ & Aggregation & PF3a \\
\hline Thrombasthenia & GPIIb-IIIa & $\begin{array}{l}\text { Prothrombinase }( \pm) \\
\text { thrombin potential }\end{array}$ & Aggregation & PF3a \\
\hline Bernard-Soulier syndrome & GPIb-IX-V & $\begin{array}{l}\text { Factor } \mathrm{XI} \text { binding, thrombin } \\
\text { binding, fibrin binding }\end{array}$ & Adhesion & SPT \\
\hline
\end{tabular}

${ }^{1}$ Department of Medicine, Columbia University College of Physicians \& Surgeons; New York, New York; ${ }^{2}$ St. Luke's-Roosevelt Hospital Center, New York, New York.

Address for correspondence and reprint requests: Harvey J. Weiss, M.D., 520 St. Nicholas Ave., Haworth, NJ 07641 (e-mail: hjw5@ columbia.edu).
Rare Bleeding Disorders; Guest Editor, Flora Peyvandi, M.D., Ph.D. Semin Thromb Hemost 2009;35:447-448. Copyright (C) 2009 by Thieme Medical Publishers, Inc., 333 Seventh Avenue, New York, NY 10001, USA. Tel: +1(212) 584-4662.

DOI 10.1055/s-0029-1226014. ISSN 0094-6176. 